\title{
Predicción del rendimiento académico en la asignatura de Matemática Básica en la unalm utilizando la técnica de Regresión Logística Binaria
}

\author{
Prediction of academic performance in the subject of Basic Mathematics in the unalm using the \\ binary Logistics Regression Technique
}

Rocío Delgado ${ }^{1}$; Mónica Gutiérrez²; Juan Dueñas ${ }^{3}$

\begin{abstract}
Resumen
El objetivo de este trabajo fue identificar un modelo de predicción del rendimiento académico de los estudiantes ingresantes en la asignatura de Matemática Básica, en la Universidad Nacional Agraria La Molina (UNALM). La muestra ( $\mathrm{n}=298)$ estuvo constituida por estudiantes que ingresaron en el 2012 II bajo la modalidad de concurso ordinario. La técnica estadística empleada fue la regresión logística binaria. El modelo resultante permitió hacer una estimación correcta del $80,5 \%$ de los casos considerando, como variables predictoras, el orden de mérito, el número de veces que postula y los puntajes en el examen de admisión (general, razonamiento matemático y razonamiento verbal).
\end{abstract}

Palabras clave: Matemática; predicción; regresión logística; rendimiento académico en Matemática.

\begin{abstract}
The aim of this study was to develop a predictive model of the academic performance of freshman students in the subject of Basic Mathematics at the UNALM (Universidad Nacional Agraria La Molina). The sample $(n=298)$ consisted of students admitted in 2012 II under the mode of Ordinary Admission Contest. The statistical method used was binary logistic regression. The resulting model allowed a correct estimation of $80,5 \%$ of the cases considering, as predictor variables, the order of merit, the number of times they postulated and the admission test scores (general, mathematical reasoning and verbal reasoning).
\end{abstract}

Keywords: Mathematics; prediction; logistic regression; academic performance in Mathematics.

\section{Introducción}

En los tres últimos años, la tasa de aprobación en la asignatura de Matemática Básica en la Universidad Nacional Agraria La Molina (UNALM) varió entre $67 \%$ y $76 \%$, situación que puede ser mejorada si se detectan los factores previos al ingreso que incidieron en el rendimiento académico y se interviene oportunamente.

Cherjovsky (2011) señaló que el rendimiento académico de los alumnos puede ser definido de diferentes formas y considerado desde distintos puntos de vista, relacionados con la forma de medir dicho rendimiento. Asimismo, indica que puede considerarse la nota obtenida en exámenes como indicador aceptable de desempeño del alumno; sin embargo, tiene el sesgo de tener que aceptar la fiabilidad de las evaluaciones y la homogeneidad o estandarización de las mismas.
Artunduaga (2008) señala que "el rendimiento académico es un indicador de eficacia y calidad educativa. Las manifestaciones de fracaso, como el bajo rendimiento académico, la repitencia y la deserción, expresan deficiencias en un sistema universitario. Las causas del fracaso estudiantil deben buscarse más allá del estudiante mismo. No es este el único responsable de su fracaso, lo es también la institución educativa".

En el rendimiento académico influyen diversos factores, unos pertenecen o son parte del mismo estudiante (endógenos) y otros pertenecen o se encuentran en el mundo circundante (exógenos). Estos factores no actúan en forma aislada, el rendimiento académico es el resultado de la acción recíproca de lo interno y lo externo (Porcel et al., 2010).

\footnotetext{
1 Docente asociada, Departamento Académico de Matemática. Universidad Nacional Agraria La Molina, Lima, Perú. Email: dare@lamolina.edu.pe

2 Docente asociada, Departamento Académico de Matemática. Universidad Nacional Agraria La Molina, Lima, Perú. Email: monig@lamolina.edu.pe

3 Docente principal, Departamento Académico de Matemática. Universidad Nacional Agraria La Molina, Lima, Perú Email: duenas@lamolina.edu.pe
} 
Con relación a las variables predictoras del rendimiento académico, Rodríguez y Gómez (2010) indican que numerosos estudios realizados acerca de la validez predictiva de las distintas pruebas de admisión, han observado una correlación positiva con el rendimiento posterior de los alumnos, identificándolas así como uno de los mejores predictores del futuro rendimiento académico.

En Colombia, Carvajal et al. (2009) encontraron como factores influyentes en el rendimiento académico de los nuevos estudiantes en la asignatura de Matemática I, el puntaje total del Instituto Colombiano para el Fomento de la Educación Superior (ICFES), el nivel de lectura literal y el nivel de pensamiento lógico abstracto, descartando factores como la edad, género, estrato y tipo de colegio y programa, entre otros.

Sobre la técnica de regresión logística utilizada en el presente estudio, Porcel et al. (2010) señalaron su coincidencia con otros autores en considerar, como una herramienta adecuada y muy poderosa, al modelo de regresión logística para indagar sobre variables categóricas de respuesta, según el especial interés del investigador, con la ventaja adicional de no requerir la normalidad estricta de los datos.

En el trabajo realizado por Delgado y Gutiérrez (2008), se examinó el rendimiento académico (clasificado en muy bajo, bajo y medio-alto) de los estudiantes ingresantes a Matemática Básica de la Universidad Nacional Agraria La Molina (UNALM), en relación con la modalidad y especialidad de ingreso; evidenciándose en diez ciclos consecutivos, la asociación entre el rendimiento académico bajo y muy bajo con la modalidad de exoneración para los primeros puestos, el rendimiento muy bajo con la modalidad de concurso ordinario y el rendimiento medioalto con la modalidad de exonerados del Centro Pre Universitario La Molina.

En la asignatura de Matemática Básica de la UNALM, impartida a los estudiantes ingresantes en la modalidad de concurso ordinario, se observó que, en los ciclos 2011 I, 2011 II y 2012 I, la tasa de desaprobación varió entre 29,9 $\%$ y 34,8 \%, situación que llevó a compartir los recursos destinados a los estudiantes del siguiente ingreso con los estudiantes desaprobados en la asignatura en mención. Con la finalidad de mejorar el desempeño de nuestros estudiantes, se hace necesario identificar al grupo con alta probabilidad de desaprobar la asignatura a fin de realizar una intervención oportuna que permita el desarrollo de habilidades y la complementación de los conocimientos previos requeridos. En ese sentido, la pregunta que orientó el presente trabajo de investigación fue: ¿Cuál es el modelo que permite predecir el rendimiento académico en la asignatura de Matemática Básica en la UNALM?

En consideración a lo expuesto, el objetivo del presente trabajo fue identificar un modelo de predicción del rendimiento académico de los estudiantes ingresantes bajo la modalidad de concurso ordinario, matriculados en Matemática Básica en la UNALM, considerando factores previos al ingreso. Por ello, se planteó la siguiente hipótesis de trabajo: el porcentaje de clasificación correcta del rendimiento académico en Matemática Básica, empleando la técnica de regresión logística binaria en relación con el rendimiento obtenido mayor al $70 \%$. Por tanto, la relevancia del presente estudio radica en que el modelo de predicción permitirá identificar la población en riesgo de desaprobar Matemática Básica con la finalidad de tomar acciones que conduzcan a incrementar la tasa de aprobación.

\section{Materiales y métodos}

El estudio es de tipo no experimental transversal y correlacional ya que se analizaron los hechos y fenómenos de la realidad después de su ocurrencia y en un momento determinado: la población de estudiantes ingresantes en el 2012 II. Para determinar la muestra no probabilística intencional, el criterio de inclusión fue considerar a los estudiantes ingresantes, en la modalidad de concurso ordinario, que se matricularon en Matemática Básica. La muestra se constituyó con $\mathrm{n}=298$ estudiantes, que corresponde al 93,7\% del total de 318 estudiantes de la modalidad mencionada (Tabla 1).

En la Tabla 2 se observa que la muestra estuvo constituida en mayor porcentaje $(82,5 \%)$ por ingresantes cuyas edades fluctúan entre 17 y 20 años de edad. Además, el 48,3\% del total de la muestra estuvo conformada por estudiantes mujeres en tanto que el 51,7\% fueron estudiantes varones.

En la Tabla 3 se aprecia que el 23,8 \% de la muestra está representada por estudiantes de Agronomía y, con relación a la cobertura, esta fue del $100 \%$ en las carreras de Meteorología, Pesquería e Industrias Alimentarias.

Tabla 1. Distribución de ingresantes 2012 II según modalidad de ingreso

\begin{tabular}{|l|c|}
\hline \multicolumn{1}{|c|}{ Modalidades de Ingreso } & $\mathrm{f}$ \\
\hline Concurso de admisión ordinario & 318 \\
\hline Centro preuniversitario & 99 \\
\hline Primeros puestos & 38 \\
\hline Exonerados profesionales & 5 \\
\hline Traslado externo & 1 \\
\hline Convenio Andrés Bello & 3 \\
\hline Promoción y desarrollo del deporte & 1 \\
\hline Total & 465 \\
\hline Fuente: Elaboración propia a partir de datos de la Oficina Académica de \\
Estudios- UNALM.
\end{tabular}


Tabla 2. Distribución de la muestra según edad y sexo

\begin{tabular}{|c|c|c|c|c|}
\hline Edad & Femenino & Masculino & Total & $\%$ \\
\hline 16 & 3 & 2 & 5 & 1,7 \\
\hline 17 & 18 & 27 & 45 & 15,1 \\
\hline 18 & 50 & 40 & 90 & 30,2 \\
\hline 19 & 34 & 34 & 68 & 22,8 \\
\hline 20 & 22 & 21 & 43 & 14,4 \\
\hline 21 & 7 & 10 & 17 & 5,7 \\
\hline 22 & 5 & 11 & 16 & 5,4 \\
\hline 23 & 0 & 1 & 1 & 0,3 \\
\hline 24 & 2 & 3 & 5 & 1,7 \\
\hline 25 & 0 & 2 & 2 & 0,7 \\
\hline 26 & 2 & 1 & 3 & 1,0 \\
\hline 29 & 0 & 1 & 1 & 0,3 \\
\hline 30 & 0 & 1 & 1 & 0,3 \\
\hline 32 & 1 & 0 & 1 & 0,3 \\
\hline Total & 144 & 154 & 298 & 100 \\
\hline
\end{tabular}

Fuente: Elaboración propia a partir de datos de la Oficina Académica de Estudios - UNALM.
El trabajo se desarrolló considerando tres etapas: en la primera, se identificaron las variables; para ello, a propuesta de los investigadores, se utilizaron 18 variables de entrada; luego, el análisis de correlación permitió la reducción a 8 variables. En la segunda etapa, se aplicó el modelo de regresión logística binaria obteniendo el modelo predictivo y, en la tercera etapa, se probó la hipótesis de trabajo considerando la capacidad predictiva del modelo propuesto.

Se recopiló información de la muestra con relación a las siguientes características: género, edad al momento de inscribirse al examen de admisión, año de egreso del colegio, tiempo fuera del colegio, ubicación y tipo de colegio, carrera profesional de ingreso, número de opción elegida en la carrera profesional a la que ingresó, número de veces que postula, tipo de preparación, orden de mérito de ingreso, promedio final en Matemática Básica, puntaje obtenido en el examen de admisión: matemática (aritmética, álgebra, geometría, trigonometría), razonamiento matemático, razonamiento verbal, física, química, biología y puntaje general de admisión.

Los datos fueron procesados a través del software estadístico IBM SPSS Statistics v 19.0 para windows, empleando el análisis de regresión logística binaria. Este método resulta útil para los casos en los que se desea predecir la presencia o ausencia de una característica o

Tabla 3. Distribución de la muestra según la carrera profesional de ingreso

\begin{tabular}{|c|c|c|c|c|}
\hline Carrera profesional & $\begin{array}{c}\text { Estudiantes } \\
\text { (A) }\end{array}$ & $\%$ & $\begin{array}{l}\text { Total ingresantes Concurso } \\
\text { Ordinario } 2012 \text { II } \\
\text { (B) }\end{array}$ & $\begin{array}{c}\text { Cobertura } \\
(\mathrm{A} / \mathrm{B})^{*} 100 \%\end{array}$ \\
\hline Agronomía & 71 & 23,8 & 74 & 95,9 \\
\hline Biología & 15 & 5,0 & 17 & 88,2 \\
\hline Ingeniería Ambiental & 12 & 4,0 & 15 & 80,0 \\
\hline Meteorología & 16 & 5,4 & 16 & 100,0 \\
\hline Ingeniería Forestal & 19 & 6,4 & 20 & 95,0 \\
\hline Economía & 16 & 5,4 & 17 & 94,1 \\
\hline Estadística e Informática & 16 & 5,4 & 17 & 94,1 \\
\hline Ingeniería en Gestión Empresarial & 16 & 5,4 & 19 & 84,2 \\
\hline Ingeniería Agrícola & 25 & 8,4 & 29 & 86,2 \\
\hline Zootecnia & 36 & 12,1 & 38 & 94,7 \\
\hline Pesquería & 29 & 9,7 & 29 & 100,0 \\
\hline Industrias Alimentarias & 27 & 9,1 & 27 & 100,0 \\
\hline Total general & 298 & 100,0 & 318 & 93,7 \\
\hline
\end{tabular}

Fuente: Elaboración propia a partir de datos de la Oficina Académica de Estudios - UNALM. 
resultado, según los valores obtenidos de un conjunto de variables predictoras. Es similar a un modelo de regresión lineal, pero está adaptado para modelos en los que la variable dependiente es dicotómica (registra solo valores 0 y 1) y no requieren probar el cumplimiento de supuestos como normalidad y homocedasticidad. Los coeficientes de regresión logística pueden utilizarse para estimar la razón de probabilidades de cada variable independiente del modelo. La regresión logística se puede aplicar a un rango más amplio de situaciones de investigación que el análisis discriminante, puesto que las variables independientes pueden ser cuantitativas o cualitativas.

La regresión logística binaria es de gran utilidad cuando se desea conocer el efecto de las variables independientes sobre la variable dependiente dicotómica. El modelo de regresión logística binaria permite relacionar la probabilidad de éxito $\mathrm{p}$ con las variables independientes, mediante el siguiente modelo lineal:

$$
\ln \left(\frac{\pi}{1-\pi}\right)=\sum_{i=1}^{m} \beta_{i} X_{i}
$$

En este modelo, p es la probabilidad de éxito de la variable dependiente binomial o dicotómica $\mathrm{Y}$ en función de las variables independientes $X_{i}$, pudiendo ser estas variables continuas o categóricas. Los coeficientes del modelo $\beta_{1}, \beta_{2}, \ldots, \beta_{p}$ son estimados por el método de máxima verosimilitud.

En la regresión logística binaria, se realiza la selección de variables siguiendo varios métodos; en el presente estudio se utilizó el método introducir para seleccionar las variables independientes en el análisis de regresión, es decir que todas las variables en estudio se incorporaron en un solo paso.

La bondad de ajuste del modelo de regresión logística se evalúa mediante la prueba de Hosmer y Lemeshow que evalúa el grado en que la probabilidad pronosticada coincide con la observada, es decir la calibración del modelo. Este estadístico es muy útil, especialmente cuando existen variables independientes continuas, como es nuestro caso, y consiste en dividir las probabilidades en deciles; luego se calcula la distribución de estudiantes exitosos y no exitosos según la ecuación hallada y los valores reales; finalmente, ambas distribuciones pronosticadas y observadas, se contrastan con la prueba $\chi 2$.

Los datos fueron analizados de la siguiente manera:

1. Se ajustó un modelo de regresión logística binaria considerando todas las variables independientes, sin interacción entre estas, es decir:

$$
\ln \left(\frac{\pi}{1-\pi}\right)=\beta_{0}+\sum_{i=1}^{12} \beta_{i} X_{i}
$$

2. Si alguno de los efectos principales $\beta i$ no es significativo, entonces es removido para ajustar un nuevo modelo. El criterio de inclusión consiste en que la variable independiente $X_{i}$ se mantiene en el modelo si $\mathrm{p}<0,05$.
3. Dada la naturaleza de las variables independientes, se puede calcular la probabilidad estimada de que ocurra éxito en el rendimiento en Matemática Básica, de la siguiente manera:

$$
\hat{p}=\frac{\exp ^{t}}{1+\exp ^{t}}
$$

donde: $t=\hat{\beta}_{0}+\hat{\beta}_{1} X_{1}+\ldots+\hat{\beta}_{i} X_{i}$

En este caso, la significación de los coeficientes del modelo se establece de acuerdo al estadístico de Wald, que es una prueba basada en el cociente entre el coeficiente de regresión y su correspondiente error estándar. Este estadístico sigue una distribución normal y permite contrastar si el coeficiente es significativamente diferente de 0 y el valor de p para ese contraste.

\section{Resultados y discusión}

Para hallar el modelo de predicción, se declararon las siguientes variables independientes:

$\mathrm{X}_{1}$ : Género $(1=$ femenino, 2 = masculino $)$.

$\mathrm{X}_{2}$ : Edad al momento de la inscripción al concurso de admisión.

$\mathrm{X}_{3}$ : Tiempo transcurrido desde que egresó del colegio (en meses).

$\mathrm{X}_{4}$ : Lugar de procedencia del estudiante ( 1 = Lima y Callao, $2=$ otras provincias de Lima, $3=$ otros departamentos).

$\mathrm{X}_{5}$ : Tipo de colegio $(1=$ público, $2=$ privado $)$

$\mathrm{X}_{6}$ : Ubicación del colegio $(1=$ Lima y Callao, $2=$ otras provincias de Lima, 3 = otros departamentos).

$\mathrm{X}_{7}$ : Número de veces que postula a la UNALM.

$\mathrm{X}_{8}$ : Tipo de preparación $(1=$ CEPREUNALM, $2=$ academia preuniversitaria, 3 = grupo de estudio, $4=$ autoestudio, $5=$ otras formas de preparación).

$\mathrm{X}_{9}$ : Carrera profesional a la que ingresa (codificación de acuerdo al código de las carreras profesionales).

$\mathrm{X}_{10}$ : Orden de preferencia de las carreras elegidas $(1=$ carrera elegida como primera opción, 2 = segunda opción, $3=$ tercera opción).

$\mathrm{X}_{11}$ : Puntaje obtenido en Matemática en el examen de admisión (promedio ponderado de Aritmética (6), Álgebra (8), Geometría (6) y Trigonometría (4)).

$\mathrm{X}_{12}$ : Puntaje obtenido en Razonamiento Matemático.

$\mathrm{X}_{13}$ : Puntaje obtenido en Razonamiento Verbal.

$\mathrm{X}_{14}$ : Puntaje obtenido en Física.

$\mathrm{X}_{15}$ : Puntaje obtenido en Química.

$\mathrm{X}_{16}$ : Puntaje obtenido en Biología.

$\mathrm{X}_{17}$ : Puntaje general de ingreso obtenido en el examen de admisión.

$\mathrm{X}_{18}$ : Orden de mérito de ingreso.

En tanto que el rendimiento académico en Matemática Básica fue considerado como variable dependiente (Y), donde $\mathrm{Y}=0$ representa no éxito (no aprobado) e $\mathrm{Y}=1$ representa éxito (aprobado). A juicio de los investigadores, 
se propone que los promedios finales obtenidos en el curso de Matemática Básica menores a once (11) sean considerados como no éxito y los promedios finales mayores o iguales a once (11) sean considerados como éxito, teniendo en cuenta que el sistema de calificación es vigesimal.

Luego, se realizó un análisis correlacional entre el rendimiento académico y las variables independientes, que permitió seleccionar las variables a emplear en la regresión logística binaria. La Tabla 4 muestra los resultados de las variables con niveles de significancia menores a 0,05 .

Tabla 4. Correlación entre el rendimiento en Matemática Básica y las variables independientes

\begin{tabular}{|l|c|c|}
\hline Variable & $\begin{array}{l}\text { Correlación } \\
\text { de Pearson }\end{array}$ & Significancia \\
\hline Ubicación del colegio & 0,144 & 0,013 \\
\hline Orden de mérito & $-0,356$ & 0,000 \\
\hline $\mathrm{N}^{\circ}$ de veces que postula & $-0,205$ & 0,000 \\
\hline Opción de ingreso & $-0,152$ & 0,009 \\
\hline $\begin{array}{l}\text { Puntaje general del } \\
\text { examen de admisión }\end{array}$ & 0,326 & 0,000 \\
\hline $\begin{array}{l}\text { Puntaje en el área de } \\
\text { matemática }\end{array}$ & 0,250 & 0,000 \\
\hline $\begin{array}{l}\text { Puntaje en el área de } \\
\text { física }\end{array}$ & 0,255 & 0,000 \\
\hline $\begin{array}{l}\text { Puntaje en el área de } \\
\text { química }\end{array}$ & 0,160 & 0,006 \\
\hline
\end{tabular}

Se procedió al análisis de regresión logística con estas variables y a juicio de los investigadores se incorporaron las variables tipo de colegio, tipo de preparación, puntaje en Razonamiento Matemático y Verbal, dado que también se buscaba conocer los efectos de estas variables sobre el modelo final. Posteriormente, se excluyeron las variables
Tabla 5. Prueba chi cuadrado

\begin{tabular}{|c|c|c|c|}
\hline Componente & Estadístico & gl & Sig. \\
\hline Paso & 132,015 & 9 &, 000 \\
\hline Bloque & 132,015 & 9 &, 000 \\
\hline Modelo & 132,015 & 9 &, 000 \\
\hline
\end{tabular}

opción de ingreso, puntaje en Física y Química por no tener significancia para el modelo $(\mathrm{p}>0,05)$.

En la Tabla 5 se muestra la prueba Chi cuadrado para evaluar la hipótesis nula de que en el modelo todos los coeficientes b son iguales a cero. El valor hallado para el modelo es $=132,015(\mathrm{p}=0,000)$, lo que indica que las variables incluidas en el modelo son estadísticamente significativas.

En la Tabla 6 se observan los resultados para la prueba realizada a fin de contrastar la hipótesis de que cada uno de los coeficientes bi es igual a cero. Entre todas las variables, el modelo mostró como significativamente diferente de cero $(\mathrm{p}<0,05)$, a las siguientes variables: número de veces que postula, puntaje en razonamiento matemático, puntaje en razonamiento verbal, puntaje general de admisión y orden de mérito de ingreso, es decir, contribuyen significativamente al modelo de predicción. Para los parámetros de dichas variables, se obtuvieron los siguientes valores estimados: $-0,556 ;-0,169 ;-0,161 ; 0,618$ y $-0,007$ respectivamente.

El parámetro estimado para la variable puntaje general de admisión $\left(b_{17}\right)$ es positivo e indica que el ratio de probabilidades (éxito / no éxito) se incrementa en 0,618 cuando el puntaje general de admisión incrementa una unidad. Para el resto de variables ocurrirá lo contrario, es decir que, por cada unidad que incrementa el valor de la variable, el ratio de probabilidades disminuirá una cantidad igual $a b$, todo ello medido en escala logarítmica. Cabe señalar que de los datos observados, los estudiantes

Tabla 6. Variables en la ecuación de regresión logística binaria

\begin{tabular}{|c|c|c|c|c|c|c|c|}
\hline Variable & Parámetro & Estimadores $b^{1}$ & E.T. & Wald $^{2}$ & g.1. & Sig. & $\operatorname{Exp}(b)$ \\
\hline Tipo de colegio & b5 & $-0,552$ & 0,316 & 3,052 & 1 & 0,081 & 0,576 \\
\hline Ubicación del colegio & b6 & 0,450 & 0,232 & 3,780 & 1 & 0,052 & 1,569 \\
\hline $\begin{array}{l}\text { Número de veces que } \\
\text { postula }\end{array}$ & b7 & $-0,556$ & 0,150 & 13,731 & 1 & 0,000 & 0,573 \\
\hline Puntaje en Matemática & b11 & 0,021 & 0,077 & 0,070 & 1 & 0,791 & 1,021 \\
\hline $\begin{array}{l}\text { Puntaje en razonamiento } \\
\text { matemático }\end{array}$ & b12 & $-0,169$ & 0,062 & 7,453 & 1 & 0,006 & 0,845 \\
\hline $\begin{array}{l}\text { Puntaje en razonamiento } \\
\text { verbal }\end{array}$ & b13 & $-0,161$ & 0,071 & 5,076 & 1 & 0,024 & 0,852 \\
\hline Puntaje general de ingreso & b17 & 0,618 & 0,168 & 13,610 & 1 & 0,000 & 1,855 \\
\hline Orden de mérito & b18 & $-0,007$ & 0,002 & 16,458 & 1 & 0,000 & 0,993 \\
\hline
\end{tabular}

E.T.: error estándar.

1: Coeficiente estimado. 2: Estadístico de Prueba. 
con puntaje general de admisión mayor o igual a 13,4 tienen mayores probabilidades de aprobar la asignatura Matemática Básica (92,3\%).

El modelo de regresión logística hallado para predecir el rendimiento en Matemática Básica fue:

$$
\ln \left(\frac{\pi}{1-\pi}\right)=-0,556 X_{7}-0,169 X_{12}-0,161 X_{13}+0,618 X_{17}-0,007 X_{18}
$$

con lo cual la probabilidad estimada para el éxito en el rendimiento académico es:

$$
\begin{gathered}
\hat{p}=\mathrm{P}(\text { rendimiento }=1)=\frac{\exp ^{t}}{1+\exp ^{t}} \\
\text { donde: } t=-0,556 X_{7}-0,169 X_{12}-0,161 X_{13}+0,618 X_{17}-0,007 X_{18}
\end{gathered}
$$

La Tabla 7 permite evaluar el ajuste del modelo de regresión, comparando los valores pronosticados con los valores observados. Se ha empleado un punto de corte de la probabilidad de Y para clasificar a los estudiantes de 0,5 , es decir, si la probabilidad calculada es menor a 0,5 , el modelo clasificó al rendimiento como no éxito, en tanto que si la probabilidad resultó mayor o igual a 0,5 se clasificó al rendimiento como éxito.

El modelo ajustado clasificó correctamente el rendimiento en Matemática Básica del 80,5\% de estudiantes ingresantes y matriculados en el 2012 II, lo cual se considera un ajuste óptimo. Es importante señalar que, del grupo de estudiantes que desaprobaron Matemática Básica, 40 de ellos, que representan el 48,2 \%, fueron clasificados correctamente, en tanto que del grupo de estudiantes que aprobaron el curso, 200, que representan el $93 \%$, fueron clasificados correctamente.

En la Fig. 1 podemos ver la representación de la capacidad de predicción del modelo. Se puede comprobar que el modelo calculó las probabilidades menores de 0,5 para los casos en los que se observó no éxito en su gran mayoría y asimismo calculó probabilidades mayores o iguales a 0,5 para los casos en donde se observó éxito en su gran mayoría, no obstante también se incluyeron valores observados como no éxito. Para evaluar la bondad de ajuste del modelo de regresión logística, se empleó la prueba de Hosmer y Lemeshow y se obtuvo un valor de $=$ $13,867(\mathrm{p}=0,085)$ lo cual indica que no existen diferencias estadísticamente significativas entre las clasificaciones observadas y predichas; lo cual permite aceptar que el modelo de regresión logístico hallado es adecuado.

Tabla 7. Clasificación de estudiantes 2012 II según rendimiento en Matemática Básica

\begin{tabular}{|c|c|c|c|}
\hline \multirow{2}{*}{$\begin{array}{c}\text { Rendimiento } \\
\text { Observado }\end{array}$} & \multicolumn{2}{|c|}{$\begin{array}{c}\text { Rendimiento } \\
\text { pronosticado }\end{array}$} & \multirow{2}{*}{$\begin{array}{c}\text { Porcentaje de } \\
\text { clasificación } \\
\text { correcta }\end{array}$} \\
\cline { 2 - 3 } & No éxito & Éxito & 48,2 \\
\hline No éxito & 40 & 43 & 93,0 \\
\hline Éxito & 15 & 200 & 80,5 \\
\hline $\begin{array}{c}\text { Porcentaje } \\
\text { Global }\end{array}$ & & & \\
\hline
\end{tabular}

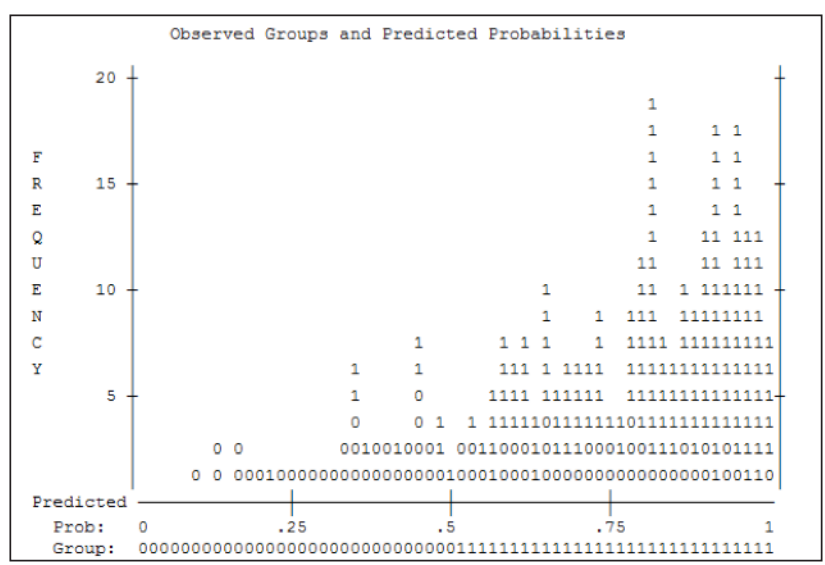

Figura 1. Clasificación de los casos observados y pronosticados

\section{Conclusiones}

Se logró formular un modelo de regresión logística binaria que, en el ciclo 2012 II, presenta una clasificación correcta del $80,5 \%$ para la predicción del rendimiento académico (aprobado o no aprobado) de estudiantes ingresantes, bajo la modalidad de concurso ordinario, matriculados en la asignatura de Matemática Básica en la Universidad Nacional Agraria La Molina. Además, se encontró que las cinco variables influyentes en la predicción del rendimiento académico fueron: el número de veces que postula a la UNALM, puntaje en razonamiento matemático, puntaje en razonamiento verbal, puntaje general obtenido en el examen de admisión y el orden de mérito.

\section{Literatura citada}

Artunduaga, M. 2008. Variables que influyen en el rendimiento académico en la universidad. Madrid, España: Departamento MIDE (Métodos de Investigación y Diagnóstico en Educación). Recuperado de http://www.ori. soa.efn.uncor.edu/?publicaciones=variables-que-influyenen-el-rendimiento-academico-en-la-universidad

Carvajal, P.; Mosquera, JC. y Artamonova, I. 2009. Modelos de predicción del rendimiento académico en matemáticas I en la Universidad Tecnológica de Pereira. Revista Scientia et Technica, 15(43): 258-263.

Cherjovsky, MR. y Loianno, M. 2011. Relación entre sistema de admisión y rendimiento académico. Revista Argentina de Educación Médica, 4(2): 55-62.

Delgado, R. y Gutiérrez, M. 2008. Rendimiento académico de estudiantes de primer ciclo en relación a la modalidad y especialidad de ingreso en la UNALM. Anales Cientificos UNALM, 69(4): 12-21.

Porcel, E.; Dapozo, G. y Lopez, M. 2010. Predicción del rendimiento académico de alumnos de primer año de la FACENA (UNNE) en función de su caracterización socioeducativa. Revista Electrónica de Investigación Educativa (REDIE), 12(2). Recuperado de <http://www. scielo.org.mx/scielo.php?script $=$ sci_arttext\&pid=S1607$40412010000200007 \& \operatorname{lng}=\mathrm{es} \& \mathrm{nrm}=\mathrm{iso}>$ 